\title{
Widespread neuroendocrine malignancy within the central nervous system: A diagnostic conundrum
}

\author{
D J Hopster, S F D Robinson, L Chadwick, J F Geddes
}

\begin{abstract}
A 75 year old female presented with a sellar tumour, and was subsequently found also to have a cauda equina tumour, a parietal dural tumour, a pontine tumour, an intradural spinal tumour, and several vertebral body tumours. Histological examination revealed a neuroendocrine tumour forming cell nests surrounded by reticulin. There was moderate nuclear pleomorphism, prominent mitoses, and focal necrosis. Immunohistochemistry showed diffuse positive staining with cytokeratins, chromogranin and 5-hydroxytryptamine, and focal positive staining with $\mathrm{S} 100$. This case is an unual and ultimately insoluble, diagnostic problem; however, the differential diagnoses include pituitary carcinoma, malignant paraganglioma, and atypical carcinoid. (F Clin Pathol 1997;50:440-442)
\end{abstract}

Keywords: neuroendocrine malignancy; carcinoid; paraganglioma

The histopathological examination of neuroendocrine tumours poses particular diagnostic problems for several reasons. One of the most frustrating of these is that the histological appearances do not correlate well with behaviour: metastasis from tumours with a bland cytological appearance is well documented, and tumours showing nuclear atypia may not behave in a malignant fashion. While neuroendocrine differentiation may be evident on light microscopy in some cases, in others immunocytochemistry or electron microscopy may be required. Another problem is that neuroendocrine tumours arise from a wide variety of sites ranging from organoid collections of endocrine
Department of Morbid Anatomy, The Royal London Hospital, E1 1BB, United Kingdom D J Hopster S F D Robinson J F Geddes

Department of Neurosurgery L Chadwick

Correspondence to: Dr Robinson

Accepted for publication 12 March 1997 Whitechapel, London the central nervous system: sella turcica, pons, cauda equina, spinal intradural, and parietal falx, together with multiple foci within vertebral bodies. All these tumours presented within months of each other, thus obscuring which, if any, was the primary site.

\section{Case report}

A 75 year old female first presented in June 1995 with symptoms and signs of hypopituitarism, together with a deterioration in visual acuity. Computed tomography (CT) revealed a lesion arising from the pituitary fossa with suprasellar extension. The appearances were consistent with a pituitary adenoma and she was treated with replacement therapy; however, no surgical procedure was undertaken at that time.

In October 1995 the patient presented with an exacerbation of lower back pain of one year's duration. A two day history of urinary retention was elicited. Examination revealed decreased sensation within dermatomes L3, L4, and L5, together with absent ankle and knee reflexes bilaterally. CT myelography revealed an intradural lesion at the level of L3-L4. This was surgically decompressed, and tissue removed for histology.

Postoperative recovery of function was good, however, her visual acuity continued to deteriorate. Ophthalmological examination suggested a bitemporal defect, probably produced by the chiasmal lesion. Magnetic resonance imaging (MRI) confirmed the previously identified sellar lesion, but also detected a central pontine lesion, a lesion apparently arising from the parietal falx, and a smaller, equivocal dural lesion nearby. In addition, an MRI of the cervical and thoracic spine showed a rounded intradural tumour at the level of $\mathrm{T} 1$ and vertebral body tumours at $\mathrm{T} 5, \mathrm{~T} 7$, and $\mathrm{T} 8$. A right pteryonal craniotomy and debulking of the chiasmatic tumour was performed and tissue was submitted for histology. The histological appearances were similar to that of the cauda equina tumour.

There was no improvement in visual acuity and further treatment was felt to be inappropriate because of the presence of widespread tumour. Of note was a complete absence of any symptoms related to other organ systems. Hormone assays were also consistently negative and there was no relevant past medical or family history. difficult if the primary site is not known or if multiple possibilities are present.

We present the results of histological and immunohistochemical examination of a neuroendocrine tumour present at five sites within 


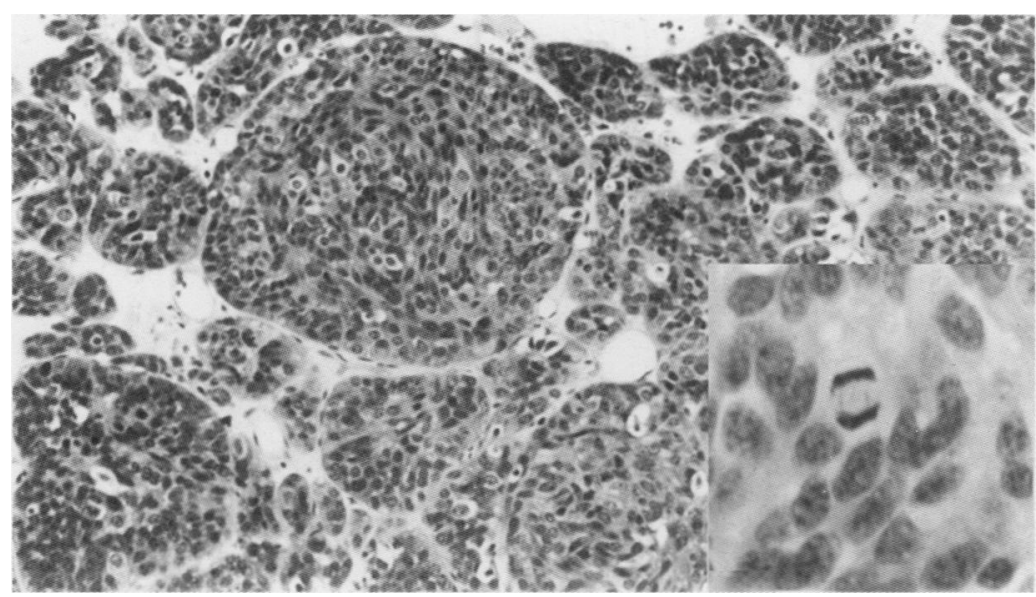

Figure 1 Low power showing tumour composed of variably sized cell nests. High power inset showing mitotic activity.
There was diffuse immunoreactivity of tumour cells with cytokeratin markers (CAM 5.2 (Becton Dickinson, UK) and MNF 116 (Dako, Denmark)) and neuroendocrine markers neuron specific enolase (NSE, Dako), NCAM (Serotec Ltd, UK), chromogranin A (Dako), and focal positive staining with anti-5hydroxytryptamine (Dako). Focal positivity for S100 was also seen within the tumour, but appeared to correspond to sustentacular cells, in that the $\mathrm{S} 100$ positive cells were situated predominantly at the edges of the cell nests (fig 2). Staining for carcinoembryonic antigen (CEA, Dako), glial fibrillary acidic protein (Dako), human melanoma clone B45 (Dako, USA), and pituitary secretory hormones (adrenocorticotrophic hormone, alpha subunit, thyroid stimulating hormone, follicle stimulating hormone, growth hormone, luteinising hormone, alpha human chorionic gonadotrophin, and prolactin) was negative.

As both biopsy specimens were small, no residual tissue was available for de novo electron microscopy. acinar formation (fig 1). Sheets of cells were also present with no specific architectural pattern. No ribbon-like or trabecular areas were seen. Foci of necrosis were present but small The cells ranged from plump and epithelioid to spindle cell in type, and had a moderate amount of eosinophilic cytoplasm. The nuclei showed moderate pleomorphism, with occasional giant forms. The nuclear chromatin had a speckled distribution and occasional single nucleoli were seen. Mitotic figures were easily identified with, at most, three mitoses per 10 high power fields.

Special stains showed reticulin encapsulation of the cell nests, but no reticulin around individual cells. Stains for mucin and glycogen were negative. A Grimelius stain showed positive cytoplasmic granularity.

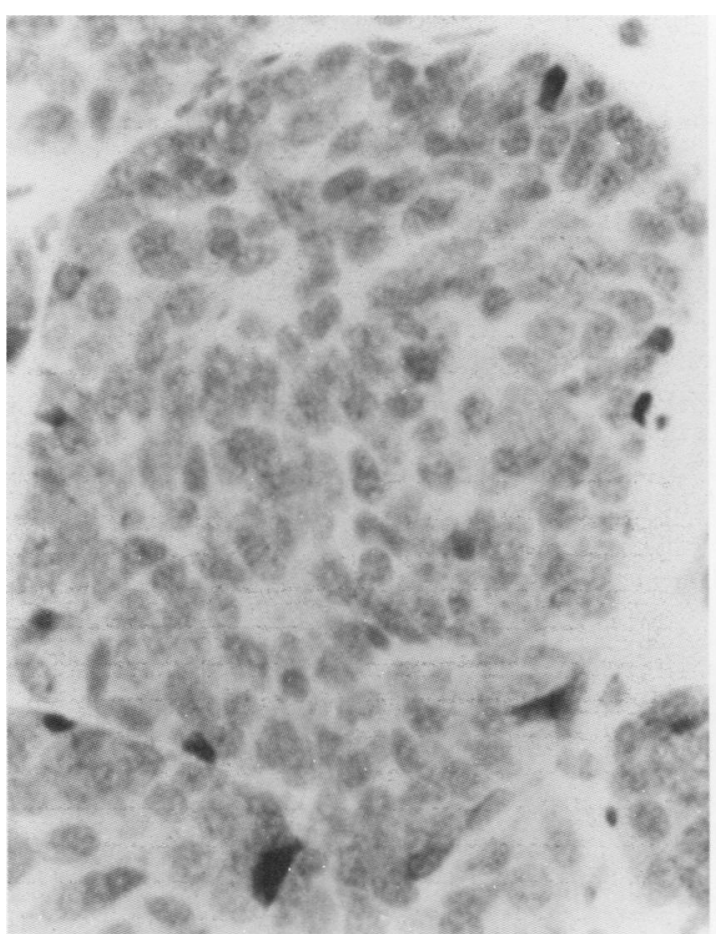

\section{Discussion}

The presence of widespread tumour precluded a diagnosis on the basis of primary site. The tumours were neuroendocrine in nature, with features that increased the likelihood of malignant behaviour. Several differential diagnoses were considered: an atypical carcinoid of unknown primary site, a malignant paraganglioma, and a pituitary carcinoma. This fascinating case posed an ultimately insoluble diagnostic conundrum, and to our knowledge no such case has been reported previously.

Neuroendocrine tumours of the diffuse endocrine system, particularly those of the lung and gastrointestinal tract, have traditionally been referred to as carcinoid tumours. Their

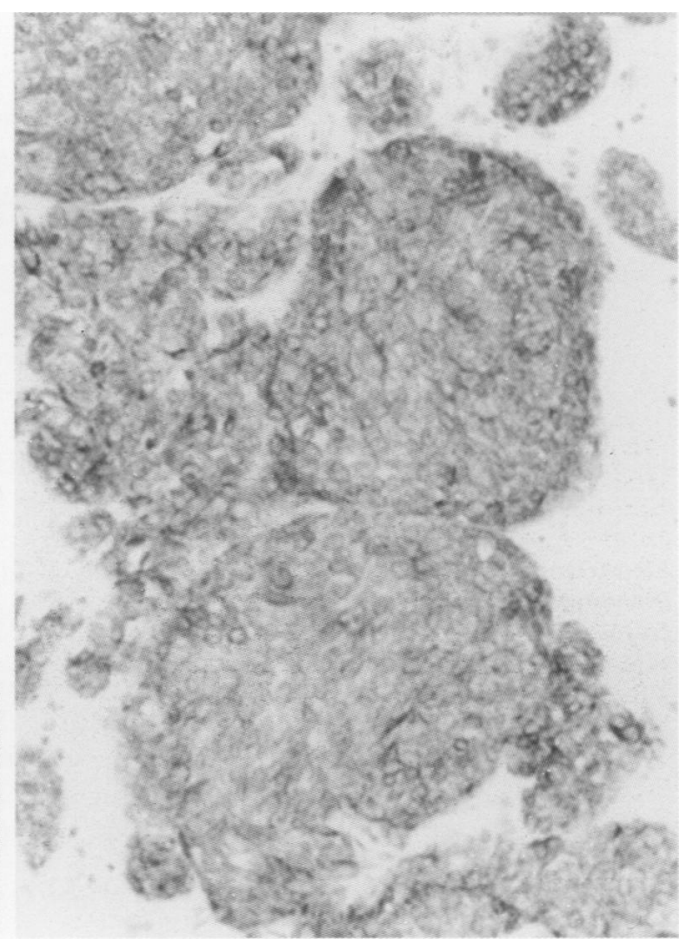

Figure 2 Left: Medium power showing cell nest with focal S100 positive cells at the periphery. Right: Low power showing diffuse positivity of tumour cells for chromogranin. 
behaviour is unpredictable; tumours with increased malignant potential, by virtue of size and cytology, being termed atypical carcinoids. In addition to positive staining with routine epithelial and neuroendocrine markers, they usually express CEA but not $\$ 100$. Although commonly presenting as a primary lesion, metastases are not unusual and silent primary tumours have been described. ${ }^{1}$ Neurological complications are present in $40 \%$ of metastatic carcinoids and true central nervous system metastases have been documented both within the brain and spinal cord. ${ }^{2}$

Primary paragangliomas of the cauda equina are well described ${ }^{3}$ and, although malignant behaviour is rare, metastatic spread has been documented. ${ }^{4}$ Nuclear atypia and necrosis are unusual features. Positive staining for cytokeratin is uncommon and has not been reported for CEA to our knowledge. The supporting sustentacular cells are classically $\$ 100$ positive, although they are not always present. ${ }^{5}$

Pituitary carcinomas are a well recognised albeit rare entity. Generalised spread has been reported $^{6}$ although central nervous system spread is more common. Metastasis to the cauda equina has been documented. ${ }^{7}$ Most carcinomas are histologically identical to adenomas; however, nuclear atypia has been recorded and said to indicate increased malignant potential. ${ }^{8}$ Cytokeratin positivity has been reported ${ }^{9}$ but is uncommon; however, the pituitary is a relatively common site for metastatic carcinoma from other sites. ${ }^{10}$

\section{Conclusion}

This widespread tumour illustrates the problem of classifying neuroendocrine tumours when there is no obvious primary site. The diagnoses of atypical carcinoid, malignant paraganglioma, and pituitary carcinoma were all considered; however, our favoured diagnosis was atypical carcinoid.

1 Nida TY, Hall WA, Glantz MJ, Clark HB. Metastatic carcinoid tumour to the orbit and brain. Neurosurgery 1992:31:949-52.

2 Patchell RA, Posner JB. Neurologic complications of carcinoid. Neurology 1986;36:745-9.

3 Sonneland PRL, Scheithauer BW, LeChago J, Crawford BG, Onofrio BM. Paraganglioma of the cauda equin region. Clinicopathologic study of 31 cases with special reference to immunocytology and ultrastructure. Cancer 1986;58:1720-35.

4 Lack EE, Cubilla AL, Woodruff JM. Paragangliomas of the head and neck region. A pathologic study of tumours from 71 patients. Hum Pathol 1979;10:191-218.

5 Brodkey JA, Brodkey JS, Watridge CB. Metastatic paraganglioma causing spinal cord compression. Spine 1995;20: glioma

6 Scheithauer BW, Randall RV, Laws ER, Kovacs KT, Horvath E, Whitaker MD. Prolactin cell carcinoma of th pituitary. Clinicopathologic, immunohistochemical, and ultrastructural study of a case with cranial and extracrania metastases. Cancer 1985;55:598-604.

7 Asai A, Matsutani M, Funada N, Takakura K. Malignant growth hormone-secreting pituitary adenoma with haematogenous dural metastasis: case report. Neurosurgery 1988 22:1091-4.

8 Challa VR, Marshall RB, Hopkins MB, Kelly DL, Civantos F. Pathobiologic study of pituitary tumours: report of 62 Fases with a review of the recent literature. Hum Pathol cases with a re
$1985 ; 16: 873-84$

9 Frost AR, Tenner S, Tenner M, Rollhauser C, Tabbara SO. ACTH-producing pituitary carcinoma presenting as the cauda equina syndrome. Arch Pathol Lab Med 1995;119. 93-6.

10 Nudleman KL, Choi B, Kusske JA. Primary pituitary carcinoma: a clinical pathological study. Neurosurgery 1985;16:90-5.
Department of Pathology, Western Infirmary, Glasgow G11 6NT, United

Kingdom

M R Stephen

K Oien

R K Ferrier

R A Burnet

Correspondence to: Dr M R Stephen.

Accepted for publication 26 February 1997

\title{
Effusion cytology of hepatocellular carcinoma with in situ hybridisation for human albumin
}

\author{
M R Stephen, K Oien, R K Ferrier, R A Burnett
}

\begin{abstract}
While the cytological features of hepatocellular carcinoma on fine needle aspiration cytology are well described, cases of hepatocellular carcinoma with malignant cells in ascitic fluid and their characteristics are not. A patient is described with cirrhosis resulting from chronic hepatitis $B$ virus infection, ascites, and hepatocellular carcinoma diagnosed by effusion cytology. The malignant cells in the effusion were shown to be positive for $\alpha$ fetoprotein using immunocytochemistry, and for human albumin using in situ hybridisation, confirming the diagnosis of hepatocellular carcinoma. Further investigations in a terminally ill patient were thus avoided.

(F Clin Pathol 1997;50:442-444)
\end{abstract}

Keywords: ascitic fluid; hepatocellular carcinoma; albumin; in situ hybridisation
Ascites is a common complication of both cirrhosis and hepatocellular carcinoma. The yield of malignant cells from hepatocellular carcinoma is generally low (approximately $10 \%$ in the series described by Falconieri et $\left.a l^{1}\right)$, and they may be more often identified in cases of hepatocellular carcinoma without cirrhosis. ${ }^{1}$ There may be difficulty distinguishing the malignant cells from reactive and atypical mesothelial cells which are commonly seen in effusions from cirrhotic patients. The immunocytochemical staining profile of hepatocellular carcinoma expressing positivity for keratins and negativity for $\mathrm{CEA}^{2}$ does not help to distinguish hepatocellular carcinoma cells from mesothelial cells. Positive staining for $\alpha$ fetoprotein, while helpful, is not specific. ${ }^{1}$ Albumin gene detection by in situ hybridisation is a highly specific aid to the confirmation of the origin of the malignant cells in an effusion. ${ }^{3}$ 